University of Nebraska - Lincoln

DigitalCommons@University of Nebraska - Lincoln

9-1996

\title{
Doubly Differential Cross Sections of Low-Energy Electrons Emitted in the Ionization of Molecular Hydrogen by Bare Carbon Ions
}

Lokesh C. Tribedi

J.R. Macdonald Laboratory, Department of Physics, Kansas State University, Manhattan, Kansas

P. Richard

J.R. Macdonald Laboratory, Department of Physics, Kansas State University, Manhattan, Kansas

D. Ling

J.R. Macdonald Laboratory, Department of Physics, Kansas State University, Manhattan, Kansas

Y. D. Wang

J.R. Macdonald Laboratory, Department of Physics, Kansas State University, Manhattan, Kansas

C. D. Lin

J.R. Macdonald Laboratory, Department of Physics, Kansas State University, Manhattan, Kansas

See next page for additional authors

Follow this and additional works at: https://digitalcommons.unl.edu/physicsrudd

Part of the Physics Commons

Tribedi, Lokesh C.; Richard, P.; Ling, D.; Wang, Y. D.; Lin, C. D.; Moshammer, R.; Kerby III, G. W.; Gealy, M. W.; and Rudd, M. Eugene, "Doubly Differential Cross Sections of Low-Energy Electrons Emitted in the Ionization of Molecular Hydrogen by Bare Carbon Ions" (1996). M. Eugene Rudd Publications. 31. https://digitalcommons.unl.edu/physicsrudd/31

This Article is brought to you for free and open access by the Research Papers in Physics and Astronomy at DigitalCommons@University of Nebraska - Lincoln. It has been accepted for inclusion in M. Eugene Rudd Publications by an authorized administrator of DigitalCommons@University of Nebraska - Lincoln. 


\section{Authors}

Lokesh C. Tribedi, P. Richard, D. Ling, Y. D. Wang, C. D. Lin, R. Moshammer, G. W. Kerby III, M. W. Gealy, and M. Eugene Rudd 


\title{
Doubly differential cross sections of low-energy electrons emitted in the ionization of molecular hydrogen by bare carbon ions
}

\author{
Lokesh C. Tribedi, ${ }^{1,}{ }^{*}$ P. Richard, ${ }^{1}$ D. Ling, ${ }^{1}$ Y. D. Wang, ${ }^{1}$ C. D. Lin, ${ }^{1}$ R. Moshammer,${ }^{2}$ G. W. Kerby III,${ }^{3}$ \\ M. W. Gealy, ${ }^{3}$ and M. E. Rudd ${ }^{3}$ \\ ${ }^{1}$ J.R. Macdonald Laboratory, Department of Physics, Kansas State University, Manhattan, Kansas 66506-2601 \\ ${ }^{2}$ Gesellschaft für Schwerionenforschung, Darmstadt, Planckstrasse 1, D-64291, Federal Republic of Germany \\ ${ }^{3}$ Department of Physics and Astronomy, University of Nebraska, Lincoln, Nebraska 68588-0111
}

(Received 25 March 1996)

\begin{abstract}
We have measured the double differential cross sections (DDCS) $\left(d^{2} \sigma / d \varepsilon_{e} d \Omega_{e}\right)$ of low-energy electron emission in the ionization of $\mathrm{H}_{2}$ bombarded by bare carbon ions of energy $30 \mathrm{MeV}$. The energy and angular distributions of the electron DDCS have been obtained for 12 different emission angles and for electron energies varying between 0.1 and $300 \mathrm{eV}$. We have also deduced the single differential and total ionization cross section from the measured DDCS. The data have been compared with the predictions of first Born approximations and the CDW-EIS (continuum distorted wave-eikonal initial state) model. The CDW-EIS model provides an excellent agreement with the data. [S1050-2947(96)10109-8]

PACS number(s): 34.50.Fa
\end{abstract}

Ionization is one of the most important reactions in highenergy ion-atom collisions. Much information on ionization dynamics has been obtained by measuring the doubly differential cross sections (DDCS) in ejected electron energy and angle. However, most of these measurements have been performed using low-charged projectiles such as electrons and protons (see Refs. [1-7] for details). Relatively little has been done using highly charged ions as projectiles. Compared to ionization by low-charged projectiles, ionization mechanisms involving highly charged ions are still not fully understood. The measurements on the DDCS of ionized electrons using highly charged bare ions can provide a test on the basic formulation of the quantum mechanical theory of ionization. Moreover, the doubly differential cross sections of the low-energy electron emission as a function of electron energy and the emission angle contain much richer information than the total or single differential cross sections and can provide a stringent test to the theoretical models.

Recently Rudd and co-workers have measured the energy and angular distributions of the electron DDCS of atomic and molecular hydrogen by low-energy (20-114 keV) protons $[8,9]$ and $\mathrm{He}^{+}$[10]. Manson et al. [4] have reported the DDCS measurements for helium bombarded by protons at energies between $300 \mathrm{keV}$ and $5 \mathrm{MeV}$. The first Born approximation (FBA) could reproduce quite well the data for 5 $\mathrm{MeV}$ protons. The FBA also gives reasonable agreement for $1 \mathrm{MeV}$ protons except some discrepancies for forward angles. At lower energies the FBA failed to explain the data both for protons $[4,8,9]$ and helium ions [10].

The low-energy electrons play a dominant role in the double differential ionization cross section although they are difficult to detect. The study of low-energy electron DDCS in single ionization of atoms, by highly charged ions (HCI), is relatively new. Only a few measurements have been re-

\footnotetext{
* On leave from Tata Institute of Fundamental Research, Homi Bhabha Road, Bombay-400005, India.
}

ported for partially stripped HCI projectiles $[13,14]$ and for bare ions [15]. However, there have been many studies on the high resolution Auger electron spectroscopy for ion-atom collisions (see Ref. [16] and the reviews by Stolterfoht $[17,18])$.

Because of the high charge of the projectile, the ejected electron spectra are strongly influenced by the two-center (projectile and target nuclei) Coulomb potential and by the postcollision interactions. Such effects cannot be adequately described by the first Born approximation even at relatively high projectile velocities (see below). At intermediate to high collision energies, the theoretical method commonly employed to incorporate the two-center effect is the CDW-EIS (continuum distorted wave-eikonal initial state) approximation of Crothers and McCann [11] (see the review by Fainstein et al. [12]). In this approximation the ionized electron is assumed to be influenced by the long-range Coulomb field of both the target and the projectile. The wave functions used in this model satisfy the correct asymptotic boundary conditions of the Coulombic three body system. On the other hand, the plane wave Born approximation often fails to provide accurate cross sections for ionization by HCI projectiles. Also the FBA calculations cannot reproduce the ECC (electron captured in continuum) cusp observed in the zerodegree electron spectroscopy [16].

Pedersen et al. [15] reported the DDCS measurements for ionization of $\mathrm{He}$ by $\mathrm{H}^{+}, \mathrm{He}^{2+}, \mathrm{C}^{6+}$, and $\mathrm{O}^{8+}$ ions of energy 1 and $1.84 \mathrm{MeV} / \mathrm{u}$. These measurements were done for electron energies between 6 and $200 \mathrm{eV}$ and for different angles. From the theoretical point of view the $\mathrm{H}_{2}$ is the simplest molecule to study. We report here the details of the energy and angular distributions of the low-energy electrons with energies between 0.1 and $300 \mathrm{eV}$ emitted in ionization of $\mathrm{H}_{2}$ in a collision with energetic fully stripped carbon ions. We also present the single differential cross sections (SDCS) derived by integrating the measured DDCS data over energies and angles. All the measured DDCS and SDCS and the total cross sections are compared with the FBA and CDWEIS calculations. 
Our theoretical treatment is based on the independent electron model which ignores electron-electron interaction. Furthermore, we simplify the molecular hydrogen target as an effective one-electron hydrogenic target with charge $Z_{\text {eff }}=1.064$, where $Z_{\text {eff }}^{2} / 2$ gives rise to the ionization potential of $\mathrm{H}_{2}$. To test our simple model, we also considered the ionization of atomic hydrogen by the same projectile. By multiplying the atomic hydrogen ionization cross sections by a factor of 2 and comparing them with cross sections obtained from the effective one-electron model that included the correct ionization potential of $\mathrm{H}_{2}$, we may assess the importance of electron-electron interaction and molecular effects that are absent from the present theory. The two calculations agree on differential cross sections for electron energies above $15 \mathrm{eV}$. Below $15 \mathrm{eV}$, the difference is about 10 $-20 \%$, with cross sections obtained from the effective oneelectron model being lower than that from twice the atomic hydrogen ionization. As shown below, our calculations are in good agreement with the present measurements with $\mathrm{H}_{2}$ target. It suggests that electron-electron interaction may indeed be negligible for electrons above $15 \mathrm{eV}$. The larger difference between the two calculations below $15 \mathrm{eV}$ may well indicate the failure of the one-electron model. The sensitivity of emission of extremely slow electrons to the use of different effective charges warrants more elaborate calculations using molecular wave functions in the future. Finally, we point out that the test of molecular target effects in the single ionization of $\mathrm{H}_{2}$ has been carried out extensively in the past few years [19]. At high collision energies, total single ionization cross sections for $\mathrm{H}_{2}$ target are essentially the same as twice the atomic hydrogen ionization cross sections. To our knowledge, such calculations have not been done for doubly differential cross sections.

Recent measurements by Krishnakumar et al. [20] have shown that for the present collision system $(30 \mathrm{MeV}$ $\mathrm{C}^{6+}+\mathrm{H}_{2}$ ) the probability for the dissociative ionization could be only about $5-7 \%$ of the total ionization probability. The ratio $(R)$ of double to single ionization cross sections is studied $[21,22]$ for similar collision systems. From these we estimate $R$ to be very small ( $\approx 3 \%$ ) for the present system and hence the most probable reaction channel would be $\mathrm{C}^{6+}+\mathrm{H}_{2} \rightarrow \mathrm{C}^{6+}+\mathrm{H}_{2}^{+}+e^{-}$. The present CDW-EIS calculations are carried out based on this reaction channel.

\section{EXPERIMENTAL DETAILS}

Bare carbon ions of energy $30 \mathrm{MeV}$ were obtained from the Tandem van de Graaff accelerator of the J. R. Macdonald Laboratory, Physics Department, Kansas State University. The energy and charge state selected beam was collimated by a four-jaw-slits arrangement and was made to pass through another aperture of diameter $2 \mathrm{~mm}$ before it collides with the target gas $\mathrm{H}_{2}$. This aperture was used to prevent the scattered beam and secondary electrons from entering the chamber. The current on the aperture was read separately and made negligible by reducing the beam dimension by the four-jaw-slits. The details of the electron spectrometer and the scattering chamber are described by Gealy et al. $[8,9]$ and Kerby [23]. The experimental setup including the spectrometer was moved from UNL to KSU for the present experiment. The collimator geometry for the beam entrance was changed slightly. A turbo molecular pump was used to evacuate the chamber to $2 \times 10^{-7}$ torr and a different MKS baratron was used to measure the gas pressure. The various tests regarding the performance of the spectrometer are similar to those described in Refs. [8,9]. A Mumetal shield was used inside the chamber to reduce the external magnetic field. A current carrying coil placed in the horizontal plane around the chamber was enough to reduce the stray magnetic field below $5 \mathrm{mG}$ in the region where the electrons travel before entering the analyzer. A hemispherical electrostatic analyzer [8,9] made of oxygen-free high-conductivity copper with inner and outer radii of 25 and $35 \mathrm{~mm}$ was used. The spherical surfaces were coated with carbon soot to reduce the secondary electron production from the copper surface due to the electron bombardment. Before entering the analyzer the electrons had to pass through a collimator made of a copper tube with two rectangular grounded apertures, one on each end. These two apertures of widths 4 and $3 \mathrm{~mm}$ mainly define the effective path-length solid-angle integral (see below). Additional apertures at entrance and the exit of the analyzer were biased with a small voltage $V_{0}$ in order to preaccelerate the electrons entering the analyzer. It was found that $V_{0}=+5 \mathrm{~V}$ was enough to improve the collection efficiency of the low-energy electrons. The energy analyzed electrons were detected by a channel electron multiplier mounted on the exit of the analyzer. The cone of the CEM was biased at $+100 \mathrm{~V}$ to help the low-energy electrons reach the detector. The earlier measurements [24] have shown that with this bias the efficiency of the channeltron is constant within $4 \%$ in the present energy range.

The spectrometer could be rotated between $15^{\circ}$ and $160^{\circ}$ and the electrons were detected at twelve different angles: $15^{\circ}, 30^{\circ}, 45^{\circ}, 60^{\circ}, 70^{\circ}, 75^{\circ}, 80^{\circ}, 90^{\circ}, 105^{\circ}, 120^{\circ}, 135^{\circ}$, and $160^{\circ}$. The data were collected in fine energy steps between 0.1 and $300 \mathrm{eV}$. Between 0.1 and $5 \mathrm{eV}$ the step size was $0.2 \mathrm{eV}$. For $\theta=70^{\circ}$ and $80^{\circ}$ the data were collected between 10 and $300 \mathrm{eV}$. The chamber was flooded with the $\mathrm{H}_{2}$ gas and the gas pressure was kept low $(0.1 \mathrm{mT})$ in order to minimize the scattering of the low-energy electrons emitted in the ionization of the target. The data were corrected for the scattering of electrons in the gas target. The correction factor was found to vary from $9.3 \%$ at $1 \mathrm{eV}$ to $9.0 \%$ at 0.1 $\mathrm{eV}$ and was less than $6 \%$ above $10 \mathrm{eV}$. These were estimated from the present geometry using the electron scattering cross section data from Golden, Bandel, and Salerno [25]. To achieve a "static"' gas pressure in the chamber a paddle was used on the top of the pump to reduce the load on the pump. For each angle the spectrum was taken with and without gas in the chamber. The data without gas were used for background subtraction.

The absolute DDCS is related to the measured electron counts $N_{e}$ (background subtracted) by the following equation:

$$
\frac{d^{2} \sigma}{d \varepsilon_{e} d \Omega_{e}}=\frac{N_{e}}{\phi \Delta W \epsilon_{\mathrm{eff}} n_{t}(l \Delta \Omega)},
$$

where $\phi$ is the number of incident particles and $\epsilon_{\text {eff }}$ is the efficiency of the detector. The quantity $n_{t}$ is the density of the gas target. The solid-angle path-length integral 
$l \Delta \Omega=\int \Omega(z) d z$ and is given by $C / \sin \theta \mathrm{cm} \mathrm{sr}$, where $C$ depends on the geometry of the apertures on the collimator in front of the analyzer. The resolution (FWHM) of the spectrometer is given by $\Delta W=R\left(\varepsilon_{e}+e V_{0}\right)$. For the present geometry of the spectrometer $R$ is about $5 \%$. The above equation can be expressed as DDCS $=\sigma_{\text {rel }} F_{n}$, where $\sigma_{\text {rel }}=N_{e} / \phi\left(\varepsilon_{e}+V_{0}\right) \sin \theta^{-1}$ and $F_{n}=\left(n_{t} \epsilon_{\text {eff }} C R\right)^{-1}$ is the normalization factor. To find the quantity $F_{n}$ experimentally, we measured the electron spectra from the ionization of $\mathrm{He}$ by $1.84 \mathrm{MeV} / \mathrm{u}$ bare $\mathrm{C}$ ions for which the cross sections are known $[12,15]$ for $\varepsilon_{e}$ between 6 and $200 \mathrm{eV}$. These measurements were carried out for three different angles, namely, $35.5^{\circ}, 90^{\circ}$, and $144.5^{\circ}$. The normalization factor was obtained by normalizing the data for $50 \mathrm{eV}$ and it was found that a constant (within 20\%) normalization factor was enough to reproduce the existing data $[12,15]$ for all the electron energies between 6 and $200 \mathrm{eV}$. The measured $F_{n}$ value was also found to be independent of the angle. It may be mentioned here that the measured $F_{n}$ was quite close (within $25 \%)$ to the $F_{n}$ estimated using the present geometry of the spectrometer and the gas pressure. However, the measured $F_{n}$ was used throughout this work for deriving the absolute cross sections. The same $F_{n}$ was used for normalizing the data below $6 \mathrm{eV}$ and above $200 \mathrm{eV}$.

The uncertainty due to the counting statistics was kept low, typically about 2-6\% except for the far backward angles for which the cross sections are very low. For these angles $\left(\theta_{e} \geqslant 120^{\circ}\right)$ the statistical uncertainty was about $5-15 \%$. The absolute uncertainties in the cross sections were typically about $25 \%$ between 5 and $100 \mathrm{eV}$ arising from the normalization procedure and counting statistics. At higher energies $\left(\varepsilon_{e} \geqslant 100 \mathrm{eV}\right)$ the cross sections being lower by a few orders of magnitude compared to that at $1 \mathrm{eV}$ cause relatively larger uncertainties (30-35\%) due to low counting statistics and background subtraction. To estimate the absolute uncertainty for electrons below $5 \mathrm{eV}$ we repeated the measurements in several different runs and found that the uncertainties could be even as large as $40-50 \%$. It is possible to have more systematic errors in the DDCS for these electrons since these can be easily deflected by any stray fields and also due to the method of background subtraction.

\section{RESULTS AND DISCUSSIONS}

\section{A. Electron doubly differential cross sections}

Electron DDCS spectra at different electron emission angles in single ionization of $\mathrm{H}_{2}$ by $30 \mathrm{MeV} \mathrm{C}^{6+}$ ions are shown in Figs. 1, 2 and 3 along with the FBA and CDW-EIS calculations.

In each case the FBA calculations are shown by dotted lines. At all angles, it is clear that there is a large discrepancy between the measurement and the first Born calculations. The largest discrepancy occurs for low-energy electrons for all the angles. For example, below $10 \mathrm{eV}$, the FBA calculations are a factor of 5 to 6 too large at forward angles. It clearly shows that the first Born approximation is not adequate to describe soft electron emissions.

It is interesting to study whether the first Born approximation is sufficient to describe electrons emitted with higher energies. In Figs. 1 - 3, we can see that the first Born results

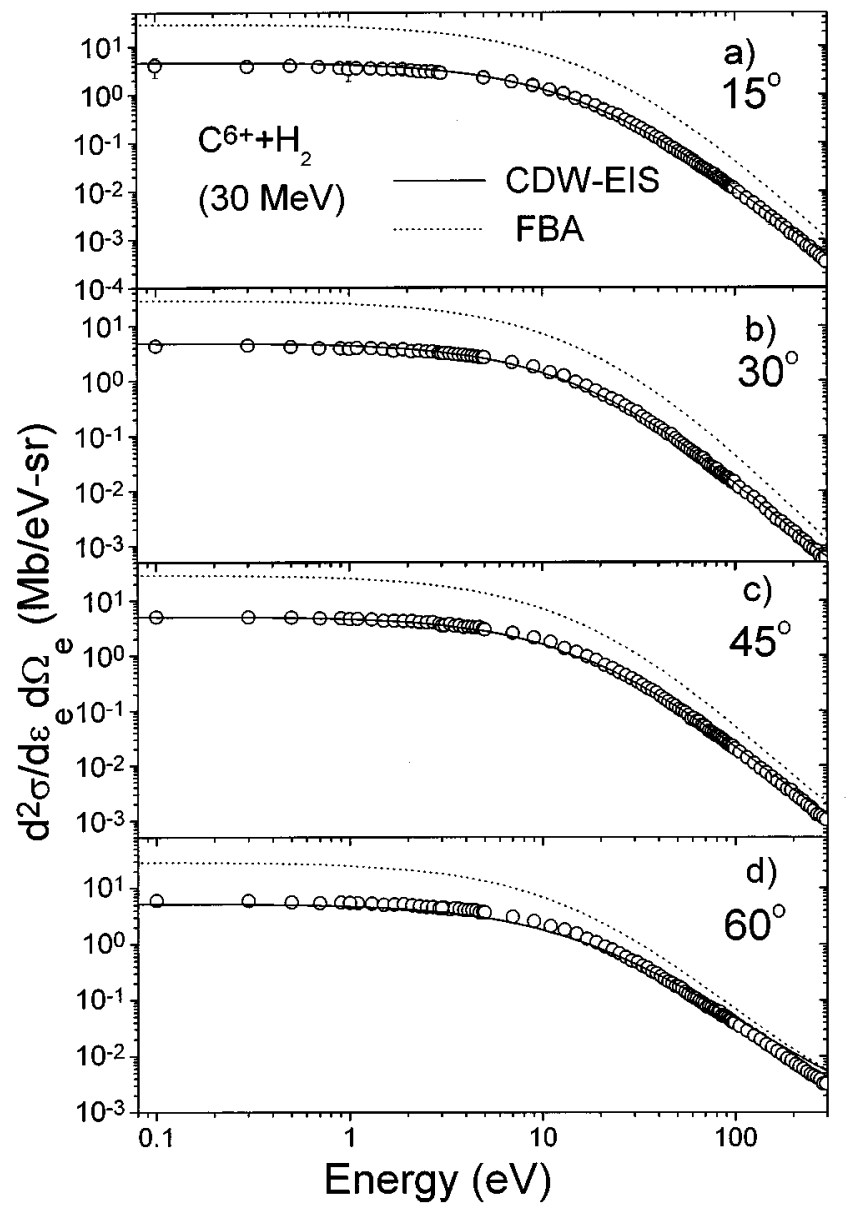

FIG. 1. (a) Double differential cross sections of electron emission for four different angles, namely, $\theta_{e}=15^{\circ}, 30^{\circ}, 45^{\circ}$, and $60^{\circ}$. The CDW-EIS and FBA calculations are shown by solid and dotted lines, respectively.

agree quite well with the data for energetic (above $150 \mathrm{eV}$ ) electrons ejected in the backward direction. However, the agreement remains poor for forward angles. This can be understood because the forward electrons are influenced more by the passing projectile and the first Born approximation does not take into account the post-collision effect. In case of ionization of $\mathrm{H}_{2}$ by protons at comparable velocities, the FBA gives much better agreement [4] with experiment. These observations show that the two-center mechanism of ionization and the post-collision effects are more important in heavy-ion impact ionization, which is not included in the first Born theory. The low-energy electrons are strongly influenced by the post-collision interaction at all emission angles, giving rise to a large deviation with the first Born theory. The fast electrons $(\geqslant 150 \mathrm{eV})$ are relatively less influenced by such interactions and hence the agreement with the FBA is reasonable.

In Figs. 1-3 we also present the CDW-EIS calculations (solid lines). There is an excellent agreement between the data and the theory for all the forward angles. The best agreement is found for angles between $15^{\circ}$ and $60^{\circ}$. It shows that the CDW-EIS theory is adequate to describe the twocenter nature of the ionization process. At backward angles, the present CDW-EIS calculations reproduce the qualitative 


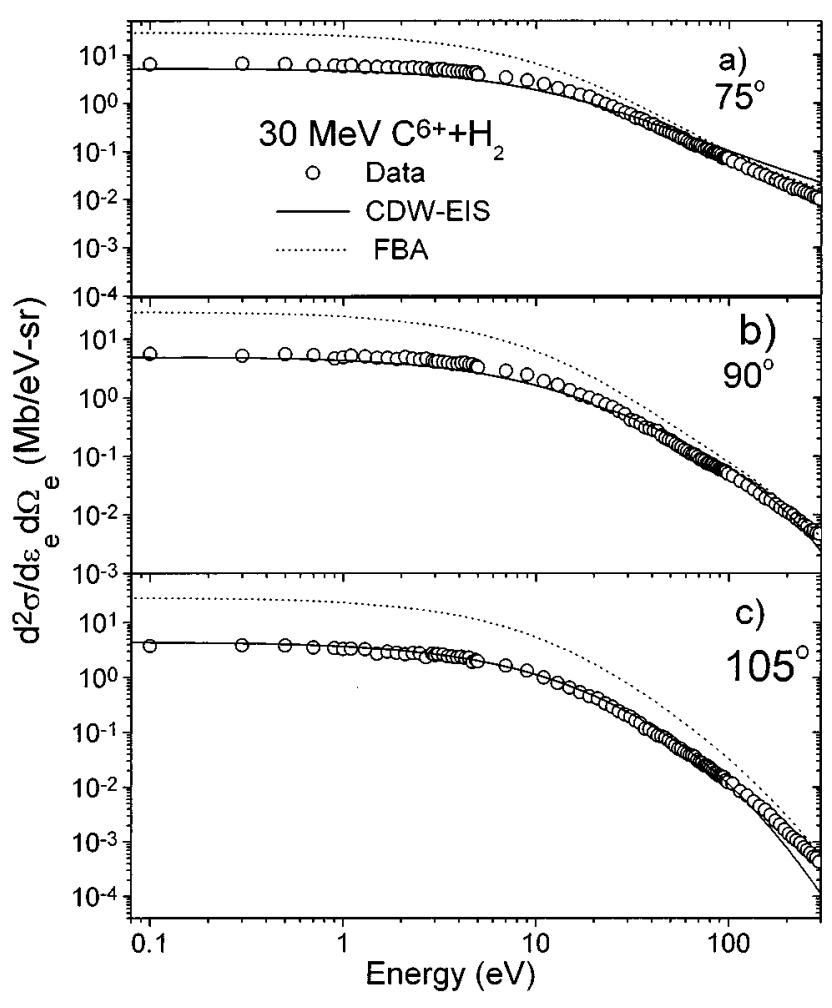

FIG. 2. Same as in Fig. 1 except for $\theta_{e}=75^{\circ}, 90^{\circ}$, and $105^{\circ}$.

behavior quite well. The best agreement is found between 10 and $100 \mathrm{eV}$. Below $10 \mathrm{eV}$ the theory overestimates the data and above $100 \mathrm{eV}$ the calculations fall below the data and the discrepancy increases at higher electron energies. In the present CDW-EIS calculations, we employed the independent electron model to treat the two-electron $\mathrm{H}_{2}$ target. The final continuum electron is described by a hydrogenic wave function with binding energy of $15.4 \mathrm{eV}$. As demonstrated by Gulyás et al. [26] a possible improvement with the DDCS at backward angles could be made if Hartree-Fock wave functions are used in both the initial and final states.

\section{B. Electron angular distribution at a fixed energy}

The comparison with theory can be studied more clearly from the angular distribution plots at fixed electron energies. The angular distributions are compared with the FBA and CDW-EIS models in Figs. 4 and 5, respectively. It is clearly seen that that the distributions peak around $75^{\circ}$ to $80^{\circ}$. For higher energy electrons the distributions gradually approach a sharp peak around $75^{\circ}-80^{\circ}$.

The electron spectrum is mainly composed of electrons arising from the soft collisions and the hard binary encounters (BE). For $\theta_{e} \leqslant 65$ the binary encounter electrons have quite high energy and do not contribute to the soft electrons in the present energy range. The BE peak, being quite wide in energy, starts contributing to the high-energy side of the soft electron spectrum for larger angles $\left(\theta_{e} \geqslant 70\right)$. The sharp peak around $\theta_{e}=75^{\circ}-80^{\circ}$ for high-energy electrons is partly due to the additional contribution of the $\mathrm{BE}$ process. For $\theta_{e}=85^{\circ}-90^{\circ}$ the $\mathrm{BE}$ peak moves into the lowest energy part of the spectrum, where the cross section for Coulomb ionization is quite large, thus giving rise to a relatively flatter

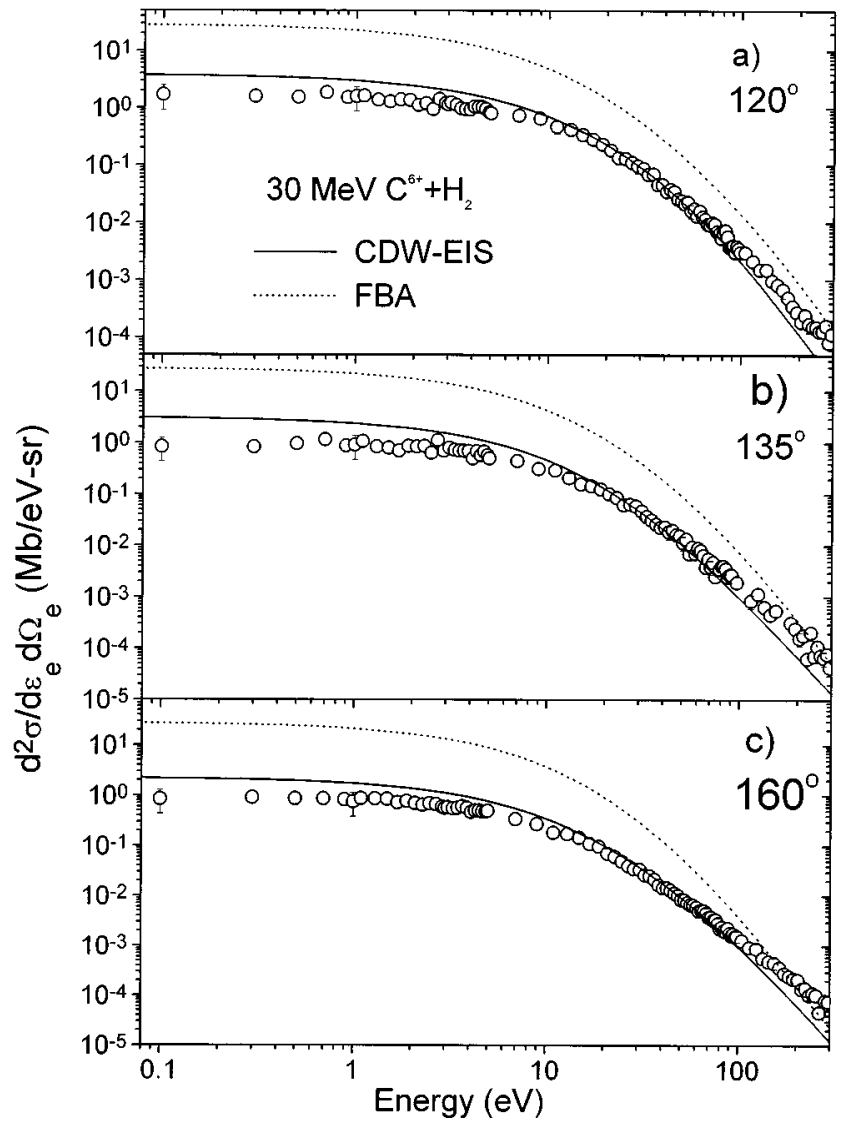

FIG. 3. Same as in Fig. 1 except for $\theta_{e}=120^{\circ}, 135^{\circ}$, and $160^{\circ}$.

angular distribution compared to the higher energy electrons. The cross sections drop rapidly in the backward direction. A similar observation was reported by Manson et al. [4] for high-energy proton impact on $\mathrm{He}$ at $5 \mathrm{MeV}$. It is worth mentioning that the angular distributions discussed above for ionization by fast protons and fast highly charged ions are quite different from those observed in ionization by low-energy protons $[4,8,9]$. For ionization of $\mathrm{H}_{2}$ and $\mathrm{H}[8,9]$ by lowenergy $(20-114 \mathrm{keV})$ protons, it was found that the electron angular distribution peaks at nearly zero degree.

We now examine the prediction of the first Born approximation on the electron's angular distribution at fixed energies. The FBA calculations predict an almost isotropic distribution for low-energy electrons [Fig. 4(a)] and largely overestimate the cross sections as mentioned before. The discrepancy in the backward direction is larger than in the forward direction. The calculations start showing a clear peak at slightly higher energies [see Fig. 4(b)]. For high-energy electrons, the discrepancy in the forward direction is comparable to that in the backward direction. It is to be noticed that for all the energies the deviation from the data is minimum around the peak, i.e., for $\theta_{e}=75^{\circ}-80^{\circ}$. Above $150 \mathrm{eV}$ [Fig. $4(\mathrm{c})]$ the discrepancy in the forward direction is much larger than that in the backward direction and the distribution is quite well reproduced by the FBA. At the highest electron energy $(295 \mathrm{eV})$, the FBA gives a good agreement for the backward angles and overestimates the cross sections in the forward directions. 


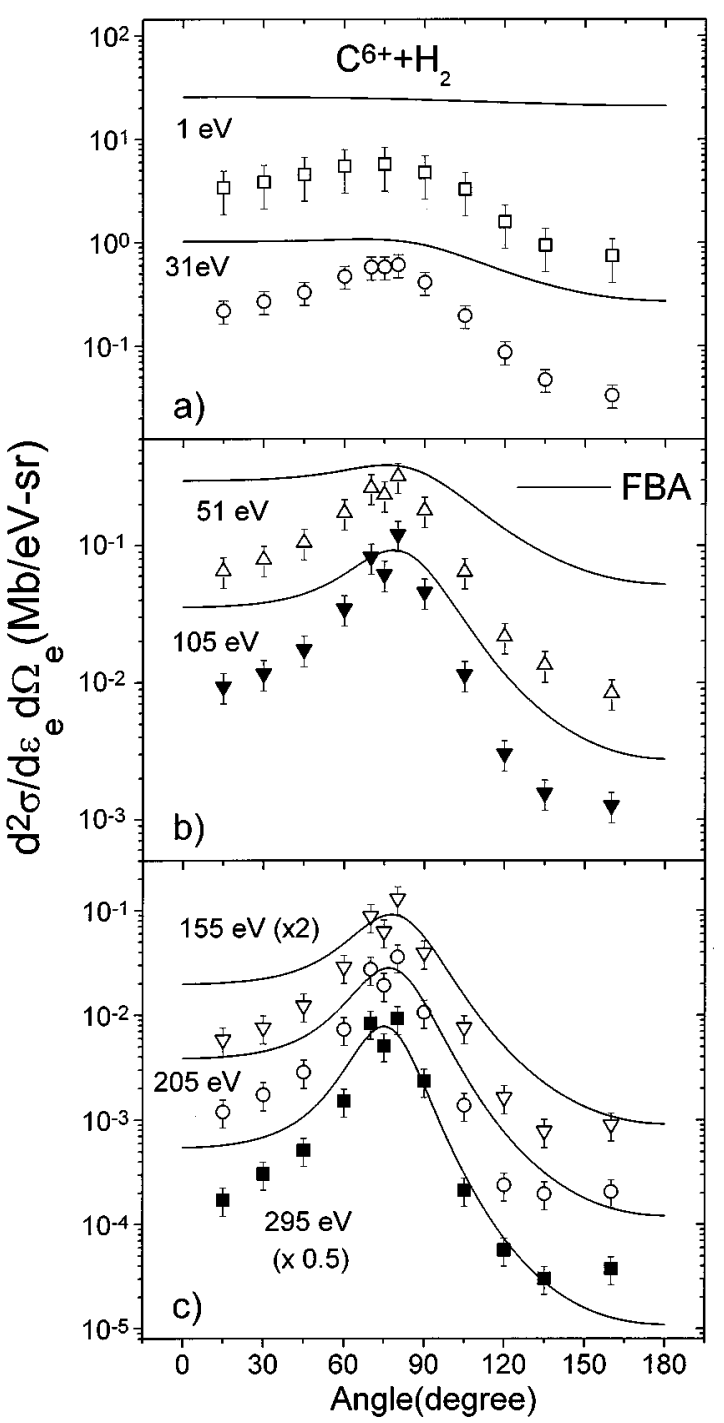

FIG. 4. Comparison of the angular distribution of electrons at a fixed energy with the FBA calculations (solid line).

The comparison of the angular distribution data with CDW-EIS is presented in Fig. 5. For $\varepsilon_{e}=0.1$ and $1 \mathrm{eV}$, the measured distributions in the forward angles agree quite well with the calculations [see Fig 5(a)]. The agreement is also good at backward angles up to $105^{\circ}$. At larger angles (e.g., $\theta_{e} \geqslant 120^{\circ}$ ), a large discrepancy is observed and for these angles the present calculations overestimate the data. For higher energies [Figs. 5(a), 5(b), and 5(c)] the theory gives an impressive agreement for all the forward angles. An excellent agreement is found for electron energies between 30 and $75 \mathrm{eV}$ for all the angles [Figs. 5(a) and 5(b)]. For higher energies the theory starts deviating from the data for backward angles. The difference between the data and the calculations increases with increasing electron energy and emission angle [see Fig. 5(c)].

\section{Single differential and total ionization cross sections}

The singly differential distributions $d \sigma / d \Omega_{e}$ and $d \sigma / d \varepsilon_{e}$ were obtained by integrating the DDCS data over electron energy or angle, respectively. The quantity $d \sigma / d \varepsilon_{e}$ is defined by

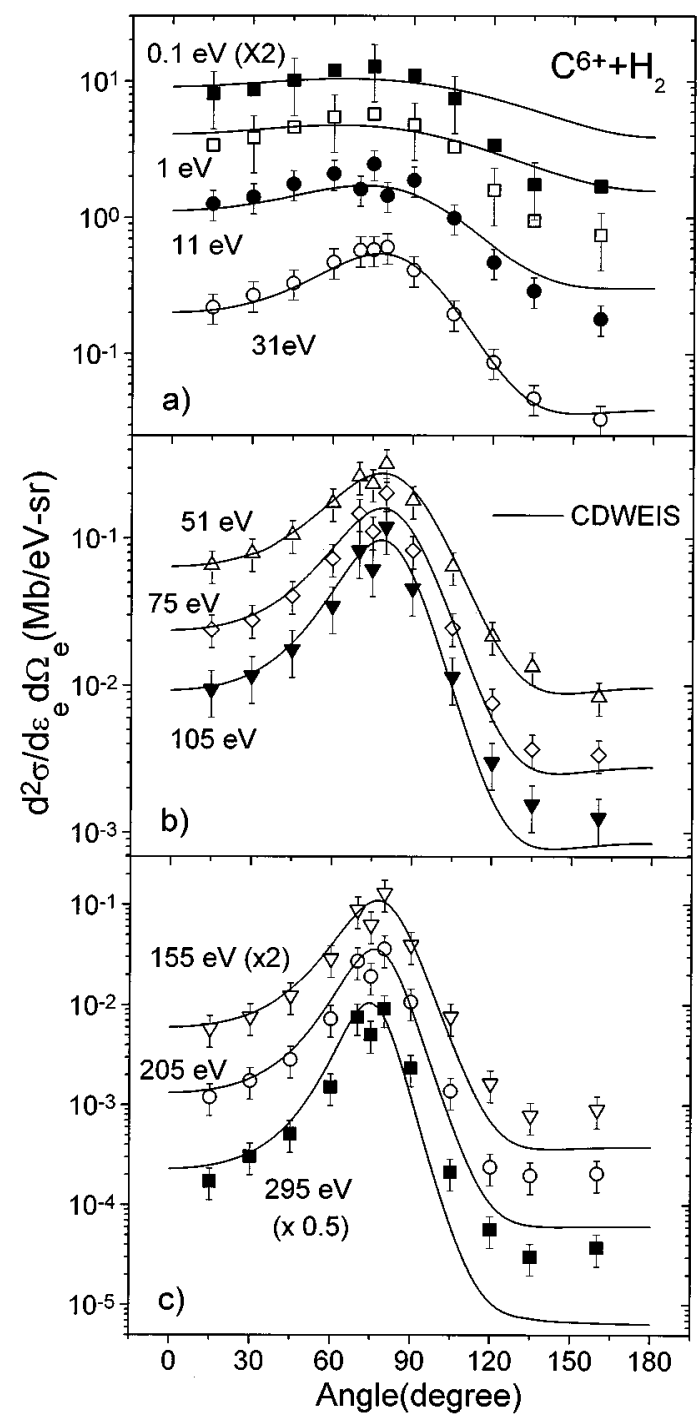

FIG. 5. Comparison of the angular distribution of electrons at a fixed energy with the CDW-EIS calculations (solid line).

$$
\frac{d \sigma}{d \varepsilon_{e}}=2 \pi \int_{\theta_{1}}^{\theta_{2}} \frac{d^{2} \sigma}{d \epsilon_{e} d \Omega_{e}} \sin \theta_{e} d \theta_{e}
$$

where $\theta_{1}=15^{\circ}$ and $\theta_{2}=160^{\circ}$. This distribution is shown in Fig. 6(a) as a function of $\varepsilon_{e}$. The CDW-EIS calculation agrees very well with the data except a small deviation above $100 \mathrm{eV}$. The FBA calculations, shown by dashed lines, overestimate the low-energy electron data by large factors and come closer to the data at higher energies. Above $150 \mathrm{eV}$ the FBA results fall slightly ( $\approx 50 \%)$ above the data and closely follow the CDW-EIS calculations.

The single differential cross sections $d \sigma / d \Omega_{e}$ are derived by integrating over the electron energies, i.e.,

$$
\frac{d \sigma}{d \Omega_{e}}=\int_{\varepsilon_{1}}^{\varepsilon_{2}} \frac{d^{2} \sigma}{d \epsilon_{e} d \Omega_{e}} d \varepsilon_{e},
$$

where $\varepsilon_{1}$ and $\varepsilon_{2}$ are 0.1 and $300 \mathrm{eV}$, respectively. The angular distributions of these cross sections are shown in Fig 6(b). The present CDW-EIS calculations explain the data 


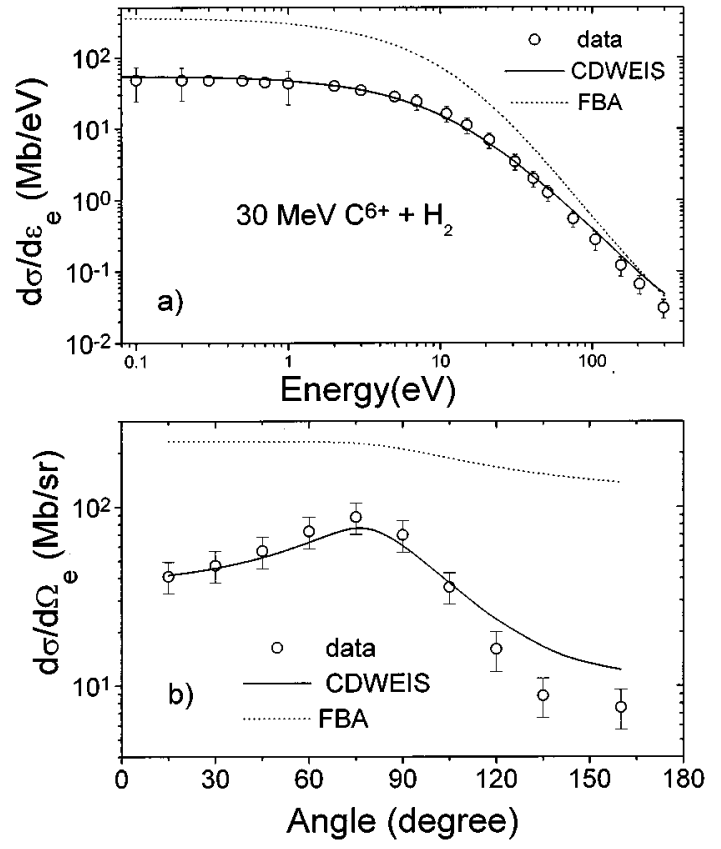

FIG. 6. (a) The single differential cross sections $\left(d \sigma / d \varepsilon_{e}\right)$ as a function of electron energy. (b) The single differential cross sections $\left(d \sigma / d \Omega_{e}\right)$ as a function of electron emission angle. The CDW-EIS and the FBA calculations are shown by solid and dotted lines, respectively, in both the figures.

very well for the forward angles whereas a large deviation is observed for backward angles. The FBA calculations overestimate the data for all the angles.

The total ionization cross section was obtained by two independent procedures. First, we integrate the angular distribution $d \sigma / d \Omega_{e}$ [Fig. 6(b)] over the electron's emission angles, i.e., $\sigma_{\text {tot }}=2 \pi \int_{\theta_{1}}^{\theta_{2}}\left(d \sigma / d \Omega_{e}\right) \sin \theta_{e} d \theta_{e}$. In a second procedure, the total cross section was obtained by integrating the electron's energy distribution in Fig. 6(a) i.e., $\sigma_{\text {tot }}=\int_{\varepsilon_{1}}^{\varepsilon_{2}}\left(d \sigma / d \varepsilon_{e}\right) d \varepsilon_{e}$. The total cross section derived from both procedures agrees with each other within $1 \%$ (arising from the numerical integration). The derived experimental total ionization cross section is $573 \mathrm{Mb}( \pm 25 \%)$ and is in excellent agreement with the CDW-EIS result of $557 \mathrm{Mb}$. The FBA prediction for the total cross section is $2488 \mathrm{Mb}$. However, it may be mentioned here that the measured $\sigma_{\text {tot }}$ does not include the cross sections for $\theta_{e}<15^{\circ}$ and $\theta_{e}>160^{\circ}$ and for $\varepsilon_{e}>300 \mathrm{eV}$. Including all the emission angles and the higher energy electrons the $\sigma_{\text {tot }}$ would be increased by about $3 \%$ which was estimated on the basis of the CDW-EIS prediction.

\section{CONCLUSIONS}

We have measured the details of the energy and angular distributions of the double differential cross sections $\left(d^{2} \sigma / d \varepsilon_{e} d \Omega_{e}\right)$ of the low-energy electrons emitted in the ionization of $\mathrm{H}_{2}$ bombarded by bare carbon ions of energy $30 \mathrm{MeV}$. The data have been presented for 12 different emission angles and for electron energies varying between 0.1 and $300 \mathrm{eV}$. The single differential distributions are also obtained by integrating the DDCS data over energies and angles. The FBA calculations are shown to strongly overestimate the low-energy data for all the angles. The calculations come closer to the data at higher energies ( $\geqslant 150 \mathrm{eV})$. The CDWEIS provides an impressive explanation of the energy and angular distributions of the DDCS although some deviations are observed for backward angles. The single differential and the total ionization cross sections are in excellent agreement with the CDW-EIS predictions. These observations are consistent with the fact that the two-center mechanism and the postcollision interactions play an important role in the heavy ion impact ionization.

Note added in proof. In a recent publication Stolterfoht et al. [27] have also shown that the CW-EIS calculations provide an excellent agreement with the $e$-DDCS data in ionization of He by energetic highly charged ions.

\section{ACKNOWLEDGMENTS}

We would like to thank C. L. Cocke and S. Hagmann for various discussions. We also thank Mark Beheren for his help in setting up the experiment. This work is supported by the Division of Chemical Sciences, National Science Foundation, Office of Basic Energy Sciences, Office of Energy Research, U.S. Department of Energy.
[1] G.B. Crooks and M.E. Rudd, Phys. Rev. Lett. 25, 1599 (1970).

[2] K.G. Harrison and M. Lucas, Phys. Lett. 33A, 149 (1970).

[3] C.B. Opal, E.C. Beaty, and W.K. Peterson, At. Data 4, 209 (1972).

[4] Steven T. Manson, L.H. Toburen, and N. Stolterfoht, Phys. Rev. A 12, 60 (1975).

[5] M.E. Rudd, L.H. Toburen, and N. Stolterfoht, At. Data Nucl. Data Tables 18, 413 (1976).

[6] M. Rudd, Y. Kim, D. Madison, and T. Gay, Rev. Mod. Phys. 64, 441 (1992).

[7] S. Suárez, C. Garibotti, W. Meckbach, and G. Bernardi, Phys. Rev. Lett. 70, 418 (1993).

[8] M.W. Gealy, G.W. Kerby III, Y.-Y. Hsu, and M.E. Rudd, Phys. Rev. A 51, 2247 (1995).
[9] G.W. Kerby III, M.W. Gealy, Y.-Y. Hsu, and M.E. Rudd, Phys. Rev. A 51, 2256 (1995).

[10] Y.-Y. Hsu, M.W. Gealy, G.W. Kerby III, and M.E. Rudd, Phys. Rev. A 53, 297 (1996); Y.-Y. Hsu, M.W. Gealy, G.W. Kerby III, M.E. Rudd, D.R. Schultz, and C.O. Reinhold, ibid. 53, 303 (1996).

[11] D.S.F. Crothers and J.F. McCann, J. Phys. B 16, 3229 (1983).

[12] P.D. Fainstein, V.H. Ponce, and R.D. Rivarola, J. Phys. B 24, 3091 (1991).

[13] R.D. DuBois, Phys. Rev. A 50, 364 (1994).

[14] C. Liao, P. Richard, S.R. Grabbe, C.P. Bhalla, T.J.M. Zouros, and S. Hagmann, Phys. Rev. A 50, 1328 (1994).

[15] J.O.P. Pedersen, P. Hvelplund, A. Petersen, and P. Fainstein, J. Phys. B 24, 4001 (1991). 
[16] D.H. Lee, P. Richard, T.J.M. Zouros, J.M. Sanders, J.L. Shinpaugh, and H. Hidmi, Phys. Rev. A 24, 97 (1990).

[17] N. Stolterfoht, Phys. Rep. 146, 315 (1987).

[18] N. Stolterfoht, J. Electron. Spectrosc. 67, 309 (1994).

[19] W.E. Meyerhof, H.-P. Hülskötter, Q. Dai, J.H. McGuire, and Y.D. Wang, Phys. Rev. A 43, 5907 (1991); Y.D. Wang, J.H. McGuire, and R.D. Rivarola, ibid. 40, 3673 (1989).

[20] E. Krishnakumar, Bhas Bapat, F.A. Rajgara, and M. Krishnamurthy, J. Phys. B 27, L777 (1994).

[21] S. Cheng, C.L. Cocke, E.Y. Kamber, C.C. Hsu, and S.L. Varghese, Phys. Rev. A 42, 214 (1990).

[22] E. Krishnakumar and F.A. Rajgara, J. Phys. B 26, 4155 (1993).
[23] G.W. Kerby III, Ph.D. thesis, University of Nebraska, 1994 (unpublished).

[24] M.A. Bolorizadeh and M.E. Rudd, Phys. Rev. A 33, 882 (1986); M.A. Bolorizadeh, Ph.D. thesis, University of Nebraska, 1984 (unpublished).

[25] D.E. Golden, H.W. Bandel, and J.A. Salerno, Phys. Rev. 140, 40 (1966).

[26] L. Gulyás, P.D. Fainstein, and A. Salin, J. Phys. B 28, 245 (1995).

[27] N. Stolterfoht, H. Platten, G. Schiwietz, D. Schneider, L. Gulyás, P. D. Fainstein, and A. Salin, Phys. Rev. A 52, 3796 (1995). 